\title{
First search for $K_{L} \rightarrow \pi^{0} \gamma$
}

N. Shimizu, ${ }^{1}$ J. K. Ahn, ${ }^{2}$ B. Beckford, ${ }^{3}$ M. Campbell, ${ }^{3}$ S. H. Chen, ${ }^{4}$ J. M. Choi, ${ }^{2}$ J. Comfort, ${ }^{5}$ K. Dona, ${ }^{3}$ M. S. Farrington, ${ }^{6}$ N. Hara, ${ }^{1}$ H. Haraguchi, ${ }^{1}$ Y. B. Hsiung, ${ }^{4}$ M. Hutcheson, ${ }^{3}$ T. Inagaki, ${ }^{7}$ M. Isoe, ${ }^{1}$ I. Kamiji, ${ }^{8}$ E. J. Kim, ${ }^{9}$ J. L. Kim, ${ }^{2, *}$ H. M. Kim, ${ }^{9}$ T. K. Komatsubara, ${ }^{7,10}$ K. Kotera, ${ }^{1}$ J. W. Lee, ${ }^{2}$ G. Y. Lim, ${ }^{7,10}$ C. Lin, ${ }^{4}$ Q. S. Lin, ${ }^{6}$ Y. Luo, ${ }^{6}$ T. Mari, ${ }^{1}$ T. Matsumura, ${ }_{11}$ D. Mcfarland,${ }^{5}$ N. A. McNeal, ${ }^{3}$ K. Miyazaki,${ }^{1}$ R. Murayama, ${ }^{1, \dagger}$ K. Nakagiri, ${ }^{8, t}$ H. Nanjo, ${ }^{1}$ H. Nishimiya, ${ }^{1}$ Y. Noichi, ${ }^{1}$ T. Nomura, ${ }^{7,10}$ T. Nunes, ${ }^{1}$ M. Ohsugi, ${ }^{1}$ H. Okuno, ${ }^{7}$ J. C. Redeker, ${ }^{6}$ K. Sato, ${ }_{1,}^{1}{ }^{\text {T. Sato, }}{ }^{7}$ Y. Sato, ${ }^{1}$ T. Shimogawa, ${ }^{12,}$ T. Shinkawa, ${ }^{11}$ S. Shinohara, ${ }^{8, \|}$ K. Shiomi, ${ }^{7,10}$ R. Shiraishi, ${ }^{1}$ S. Su, ${ }^{3}$ Y. Sugiyama, ${ }^{1, \$}$ S. Suzuki, ${ }^{12}$ Y. Tajima, ${ }^{13}$ M. Taylor, ${ }^{3}$ M. Tecchio, ${ }^{3}$ M. Togawa, ${ }^{1, \$}$ T. Toyoda, ${ }^{1}$ Y. C. Tung, ${ }^{6,}$ Q. H. Vuong, ${ }^{1}$ Y. W. Wah, ${ }^{6}$ H. Watanabe, ${ }^{7,10}$ T. Yamanaka, and H. Y. Yoshida ${ }^{13}$

\section{(KOTO Collaboration)}

\author{
${ }^{1}$ Department of Physics, Osaka University, Toyonaka, Osaka 560-0043, Japan \\ ${ }^{2}$ Department of Physics, Korea University, Seoul 02841, Republic of Korea \\ ${ }^{3}$ Department of Physics, University of Michigan, Ann Arbor, Michigan 48109, USA \\ ${ }^{4}$ Department of Physics, National Taiwan University, Taipei, Taiwan 10617, Republic of China \\ ${ }^{5}$ Department of Physics, Arizona State University, Tempe, Arizona 85287, USA \\ ${ }^{6}$ Enrico Fermi Institute, University of Chicago, Chicago, Illinois 60637, USA \\ ${ }^{7}$ Institute of Particle and Nuclear Studies, High Energy Accelerator Research Organization (KEK), \\ Tsukuba, Ibaraki 305-0801, Japan \\ ${ }^{8}$ Department of Physics, Kyoto University, Kyoto 606-8502, Japan \\ ${ }^{9}$ Division of Science Education, Jeonbuk National University, Jeonju 54896, Republic of Korea \\ ${ }^{10}$ J-PARC Center, Tokai, Ibaraki 319-1195, Japan \\ ${ }^{11}$ Department of Applied Physics, National Defense Academy, Kanagawa 239-8686, Japan \\ ${ }^{12}$ Department of Physics, Saga University, Saga 840-8502, Japan \\ ${ }^{13}$ Department of Physics, Yamagata University, Yamagata 990-8560, Japan
}

(Received 29 June 2020; accepted 8 September 2020; published 28 September 2020)

We report the first search for the $K_{L} \rightarrow \pi^{0} \gamma$ decay, which is forbidden by Lorentz invariance, using the data from 2016 to 2018 at the J-PARC KOTO experiment. With a single event sensitivity of $\left(7.1 \pm 0.3_{\text {stat }} \pm 1.6_{\text {syst }}\right) \times 10^{-8}$, no candidate event was observed in the signal region. The upper limit on the branching fraction was set to be $1.7 \times 10^{-7}$ at the $90 \%$ confidence level.

DOI: $10.1103 /$ PhysRevD.102.051103

The $K_{L} \rightarrow \pi^{0} \gamma$ decay is forbidden by the conservation of angular momentum. In the $K_{L}$ rest frame, the spin of a massless photon must be polarized along the decay axis,

*Present address: Jeonbuk National University, Jeonju 54896, Republic of Korea.

Present address: RIKEN Cluster for Pioneering Research, RIKEN, Wako 351-0198, Japan.

${ }^{\star}$ Present address: KEK, Tsukuba, Ibaraki 305-0801, Japan.

${ }^{\S}$ Present address: Institute for Space-Earth Environmental Research, Nagoya University, Nagoya, Aichi 464-8601, Japan.

"Present address: Department of Physics, Osaka University, Toyonaka, Osaka 560-0043, Japan.

Present address: National Taiwan University, Taipei, Taiwan.

Published by the American Physical Society under the terms of the Creative Commons Attribution 4.0 International license. Further distribution of this work must maintain attribution to the author(s) and the published article's title, journal citation, and DOI. Funded by SCOAP ${ }^{3}$. but the back-to-back configuration of two-body decays does not allow the parallel component of the orbital angular momentum. In the broader context, $K_{L} \rightarrow \pi^{0} \gamma$ threatens Lorentz invariance and gauge invariance [1]. Such restrictions on $K_{L} \rightarrow \pi^{0} \gamma$ provide the opportunity to search for new physics beyond the standard model (SM). In particular, as Ref. [2] suggests, similarly to experiments such as ones using optical resonators $[3,4]$, Lorentz invariance should be tested in short distances. Several scenarios predict a finite rate of the $K_{L} \rightarrow \pi^{0} \gamma$ decay [1,5]. Using charged kaons, the E949 experiment at BNL searched for the $K^{+} \rightarrow \pi^{+} \gamma$ decay and set an upper limit on the branching fraction to be $2.3 \times 10^{-9}$ [6] at the $90 \%$ confidence level (C.L.); no measurements have been made for neutral kaons.

The KOTO experiment is being carried out using the $30 \mathrm{GeV}$ main ring accelerator at J-PARC in Ibaraki, Japan. A $K_{L}$ beam was produced by protons hitting a gold target, and was transported into the KOTO detector at an angle of 
$16^{\circ}$ from the primary beam [7]. Photons in the beam were removed by a 35-mm-thick lead plate placed in the upstream, and charged particles were removed by a sweeping magnet. The solid angle of the neutral beam after a collimation was $7.8 \mu \mathrm{sr}$, and the size was $8 \times 8 \mathrm{~cm}^{2}$ at $20 \mathrm{~m}$ downstream from the target. At the exit of the beam line, the peak of the $K_{L}$ momentum distribution was $1.4 \mathrm{GeV} / c$. The $K_{L}$ incident rate to the KOTO detector was measured to be $7 \mathrm{MHz}$ at a beam power of $50 \mathrm{~kW}$, based on the measured $K_{L} \rightarrow 2 \pi^{0}$ and $K_{L} \rightarrow 3 \pi^{0}$ decays (this corresponded to $2 \times 10^{-7} K_{L}$ per proton on target).

The primary purpose of the KOTO experiment is to study the $C P$-violating $K_{L} \rightarrow \pi^{0} \nu \bar{\nu}$ decay, which is suppressed in the SM, and the branching fraction is predicted to be $(3.0 \pm 0.3) \times 10^{-11}$ [8]. The signature of $K_{L} \rightarrow \pi^{0} \nu \bar{\nu}$ is $2 \gamma+$ nothing; hence the KOTO detector consists of a finegrained electromagnetic calorimeter and hermetic veto counters surrounding the decay volume. Thus, the apparatus is ideal to search for the $K_{L} \rightarrow \pi^{0} \gamma$ decay.

Figure 1 shows the sectional view of the KOTO detector, in which the $z$ axis is in the center of the beam line. The decay volume was kept in vacuum of $10^{-5} \mathrm{~Pa}$ to suppress interactions between beam neutrons and residual gas. An electromagnetic calorimeter (CSI), consisting of 2716 50-cm-long undoped cesium iodide crystals, measured the energies and hit positions of incident photons from $K_{L}$ decays. The central and outer regions of CSI were made of $2.5 \times 2.5 \mathrm{~cm}^{2}$ and $5.0 \times 5.0 \mathrm{~cm}^{2}$ crystals in cross section, respectively $[9,10]$. The crystals were stacked inside a 1.9 -m-diameter cylinder, leaving a central $20 \times 20 \mathrm{~cm}^{2}$ hole for the beam path. The FB, MB, and IB [11] were leadscintillator sandwich counters, hermetically covering the decay volume to veto extra particles from $K_{L}$ decays. The inner surfaces of IB, MB, and the beam hole of CSI were covered with plastic scintillators (their thicknesses were 5, 10 , and $3 \mathrm{~mm}$, respectively) to veto charged particles. Two layers of 3-mm-thick plastic scintillation counters (CV) [12] were placed upstream of CSI to veto charged particles. To veto charged particles escaping through the beam hole of CSI, three layers of wire chambers were placed downstream of CSI in the beam (newBHCV). To veto photons passing through the beam hole, four sets of collar-shaped undoped cesium iodide counters were installed in CSI (CCO3) and downstream of CSI (CC04, CC05, and CC06). To veto photons passing through the beam hole, sixteen modules, each made of lead and aerogel (BHPV) [13], and four modules, each made of lead and acrylic plates (BHGC), were placed downstream of CSI. To veto particles going upstream, a counter made of undoped cesium iodide crystals (NCC) was placed inside of FB. The waveform of the signal from all the detector components was recorded with either 125 or $500 \mathrm{MHz}$ digitizers. Details of the KOTO detector are available in Refs. [14,15].

In this analysis, we used data taken in the periods of from May to June 2016, from May to July 2017, and from January to February 2018 with the proton beam power of $42-50 \mathrm{~kW}$, corresponding to $2.8 \times 10^{18}$ protons on target in total. The trigger required an energy deposit of $>550 \mathrm{MeV}$ in CSI with no coincident signals in IB, MB, $\mathrm{CC} 03, \mathrm{CC} 04, \mathrm{CC} 05, \mathrm{CC06}, \mathrm{CV}$, and NCC. The on-line energy thresholds for the veto counters were set sufficiently higher than those used in the off-line analysis to avoid acceptance loss. With CSI, the number of clusters was calculated on-line. A cluster is defined as a collection of contiguous crystals with energies deposited larger than 22 and $44 \mathrm{MeV}$ for the small and large crystals, respectively. The data with exactly three clusters in CSI was used to search for the $K_{L} \rightarrow \pi^{0} \gamma$ decay. Details of data acquisition system are available in Ref. [16].

Candidates for $K_{L} \rightarrow \pi^{0} \gamma$ were required to have exactly three clusters, $\gamma_{0}, \gamma_{1}$, and $\gamma_{2}$ (hereafter, 1,2 are indices of two $\gamma$ 's from $\pi^{0}$ decay and 0 is for the other $\gamma$ ) in CSI. Each cluster was reconstructed by integrating all adjoining crystals located within $70 \mathrm{~mm}$ and with the deposited energy larger than $3 \mathrm{MeV}$ [17]. The cluster energy was defined as the sum of all the energy deposits of the crystals in the cluster. The cluster timing and $x y$ position were defined as energyweighted averages of the timings and crystal locations, respectively. All the cluster timings were required to be within $10 \mathrm{~ns}$ of each other. The clusters were required to be outside the beam hole region: $\max \{|x|,|y|\}>150 \mathrm{~mm}$.

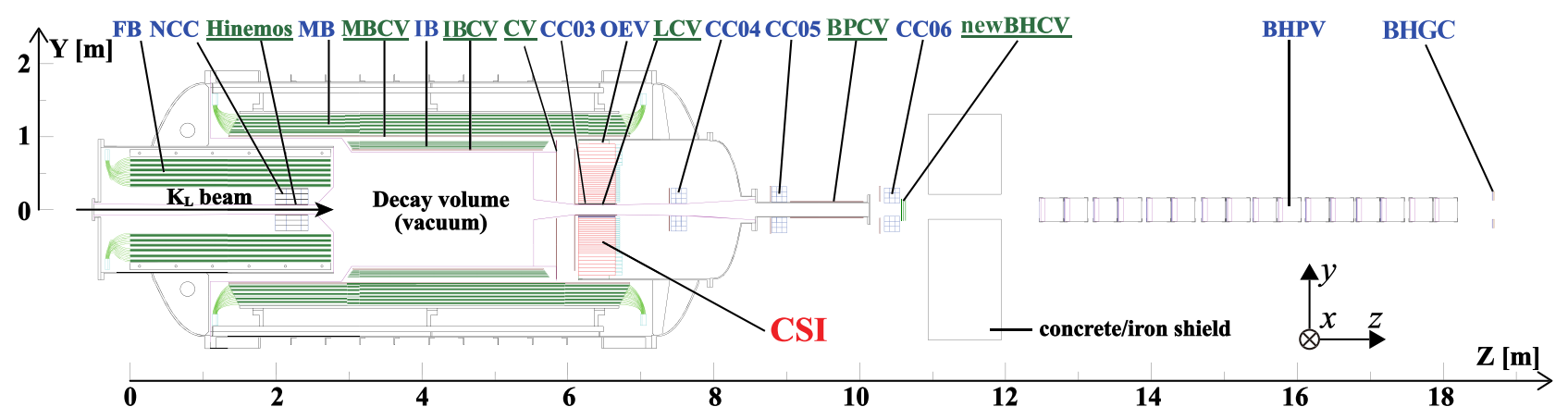

FIG. 1. Sectional view of the KOTO detector. The $K_{L}$ beam, pointing in the $+z$ direction in the figure, was transported from the left side. The names without (with) underline were for neutral (charged) particles. The origin of the $z$ axis is the upstream edge of FB. 
The decay vertex position of the $\pi^{0}$ along the beam, $z=z_{\mathrm{vtx}}^{\pi^{0}}$, was first reconstructed assuming that two of the three clusters were from the $\pi^{0}$, the decay position was along the $z$ axis, and the invariant mass, $M_{\gamma_{1} \gamma_{2}}$, was equal to the nominal $\pi^{0}$ mass. Of the three photon combinations, the one with the smallest absolute magnitude of two vertex displacement, $\Delta z_{\mathrm{vtx}}=z_{\mathrm{vtx}}^{\pi^{0}}-z_{\mathrm{vtx}}^{K_{L}}$, was selected, where $z_{\mathrm{vtx}}^{K_{L}}$ was calculated by assuming that the invariant mass of all three $\gamma$ 's equals the nominal mass of $K_{L}$. The four-momenta of the three $\gamma$ 's were then reconstructed assuming that they are produced at $z_{\mathrm{vtx}}^{\pi^{0}}$. The optimization of selection criteria (cuts) and estimation of acceptances were based on the Monte Carlo (MC) simulation using the GEANT4 package [18-20]. To reflect the real beam-related activities, the MC events were further overlaid with random trigger data taken during physics data collection.

To avoid bias, we adopted a blind analysis technique: the signal region (SR) had been defined in the two-dimensional space of $\left(z_{\mathrm{vtx}}^{\pi^{0}}, M_{\pi^{0} \gamma}\right)$, and the selection criteria were determined using a dataset with all the events in the SR removed. To gain the largest possible efficiency for $K_{L} \rightarrow \pi^{0} \gamma$ while suppressing the background contribution, the SR was defined to be $1500 \mathrm{~mm}<z_{\mathrm{vtx}}^{\pi^{0}}<3500 \mathrm{~mm}$, and $490 \mathrm{MeV}<$ $M_{\pi^{0} \gamma}<520 \mathrm{MeV}$. The range of the decay vertex position was determined to be more upstream than that of $K_{L} \rightarrow \pi^{0} \nu \bar{\nu}$ analysis (3000 $\mathrm{mm}<z_{\mathrm{vtx}}^{\pi^{0}}<5000 \mathrm{~mm}$ ), because the $K_{L} \rightarrow$ $2 \pi^{0}$ decay contributes to the downstream region. The sideband regions were used as control regions (CRs). The region referred to as CR2, defined as $3500 \mathrm{~mm}<z_{\mathrm{vtx}}^{\pi^{0}}<6200 \mathrm{~mm}$ and $400 \mathrm{MeV}<M_{\pi^{0} \gamma}<490 \mathrm{MeV}$, dominated by $K_{L} \rightarrow$ $2 \pi^{0}$ decays, was used to calculate the $K_{L}$ yield.

The shape of each cluster in the $x-y$ plane was required to be consistent with the shape of electromagnetic shower from a single photon obtained by the MC simulation. The MC template of the nominal energy deposits and their standard deviations in crystals was prepared as a function of the incident angle of $\gamma$ and the observed cluster energy, and was used to compute a $\chi^{2}$ value (shape $\chi^{2}$ ). We required all the $\gamma$ cluster candidates to satisfy $\chi^{2}<5$.0. These requirements discriminated a hadronic cluster due to neutrons or a fusion cluster from photon overlaps in close proximity. To further remove clusters produced by neutrons, a neural network technique (NN) [21] was used to distinguish $\gamma$ clusters from neutron clusters based on the information on two-dimensional cluster shape, relative energies of crystals, energy-weighted $x y$ position of the cluster, timings of the observed signals in crystals, and the $\gamma$ 's incident angle. All the $\gamma$ 's were required to have a likelihood of more than 0.8 , which corresponds to $90 \%$ efficiency for $\gamma$ 's and $\times 33$ reduction for neutrons.

To suppress other $K_{L}$ decays, we required no in-time signals in the veto counters above each threshold. In particular, we imposed stringent energy thresholds of
$1 \mathrm{MeV}$ in the three barrel counters (FB, $\mathrm{MB}$, and $\mathrm{IB}$ ) and NCC. After imposing all the veto cuts, the $K_{L} \rightarrow 2 \pi^{0}$ decay was the largest contribution of all the background sources. This decay mode could be a background if a photon with a small energy was undetected by the veto counters or two of the four clusters in CSI fused. To suppress this contribution, the two vertex displacement was required to satisfy $-100 \mathrm{~mm}<\Delta z_{\mathrm{vtx}}<200 \mathrm{~mm}$ as shown in Fig. 2(a). Furthermore, the minimum photon energy of three $\gamma$ 's $\left(E_{\gamma}^{\mathrm{min}}\right)$ was required to be larger than $600 \mathrm{MeV}$, as shown in Fig. 2(b). The $K_{L}$ momentum was calculated as a sum of momenta of $\gamma_{0}, \gamma_{1}$, and $\gamma_{2}$, and its transverse momentum and polar angle with respect to the $z$ axis were required to be less than $100 \mathrm{MeV} / c$ and $4^{\circ}$, respectively ( $K_{L}$ direction cuts). Figures 3(a) and 3(b) show the reconstructed mass $\left(M_{\pi^{0} \gamma}\right)$ versus $\pi^{0}$ decay vertex $\left(z_{\mathrm{vtx}}^{\pi^{0}}\right)$ plots after imposing the cuts described above for the $K_{L} \rightarrow$ $\pi^{0} \gamma$ decay and other $K_{L}$ decays generated by MC, respectively. A summary of the estimated acceptances for the $K_{L} \rightarrow \pi^{0} \gamma, K_{L} \rightarrow 2 \pi^{0}$, and $K_{L} \rightarrow 3 \pi^{0}$ decays at each step by the MC samples is shown in Table I. From the indices of 1 to 10 , the accumulated acceptances are shown, whereas for 11 and 12, cuts of 1 to 8 are included.

Table II summarizes the expected number of background events in the SR. The contribution of the $K_{L} \rightarrow 2 \pi^{0}$ events in the SR was estimated to be

$$
N_{\mathrm{obs}}^{\mathrm{CR} 2} p_{2 \pi^{0}}^{\mathrm{CR} 2} \times \frac{N_{\mathrm{MC}}^{\mathrm{SR}}}{N_{\mathrm{MC}}^{\mathrm{CR} 2}}
$$

where $N_{\mathrm{obs}}^{\mathrm{CR} 2}=528$ is the number of the observed events in the CR2 region with an energy threshold for photons of $E_{\gamma}^{\min }>300 \mathrm{MeV}, N_{\mathrm{MC}}^{\mathrm{SR}}\left(N_{\mathrm{MC}}^{\mathrm{CR} 2}\right)$ is the number of events in the corresponding region with an energy threshold of $E_{\gamma}^{\min }>600 \mathrm{MeV}(300 \mathrm{MeV})$ estimated by the MC simulation, and $p_{2 \pi^{0}}^{\mathrm{CR} 2}=97 \%$ is the purity of the $K_{L} \rightarrow 2 \pi^{0}$ decay in the CR2.

The number of $K_{L} \rightarrow 3 \pi^{0}$ events in the SR was estimated based on another control region dominated by the $K_{L} \rightarrow 3 \pi^{0}$ decays, $M_{01}^{2}+M_{02}^{2}>(490 \mathrm{MeV})^{2}$, where $M_{i j}^{2}=\left(p_{i}+p_{j}\right)^{2}$, and $p_{i}(i=0,1,2)$ are the four-vectors of the three $\gamma$ 's [23]. Using the number of events in this region $\left(N_{\mathrm{obs}}^{\mathrm{CR}}=108\right)$ and $N_{\mathrm{MC}}^{\mathrm{SR}} / N_{\mathrm{MC}}^{\mathrm{CR}}<4.3 \times 10^{-3}$ at $68 \%$ C.L., we estimated the number of events in the SR to be less than 0.5 at the $68 \%$ C.L.

If the $K_{L} \rightarrow 2 \gamma$ decay was coincident with an accidental hit in CSI, it could become a background. We generated the MC samples of the $K_{L} \rightarrow 2 \gamma$ decay, corresponding to 18 times the experimental data, and found that no events satisfied the cuts.

Another type of background was the $\pi^{0}$ production by an interaction of beam halo neutrons in NCC, where two photons from the $\pi^{0}$ decay entered CSI with an additional 


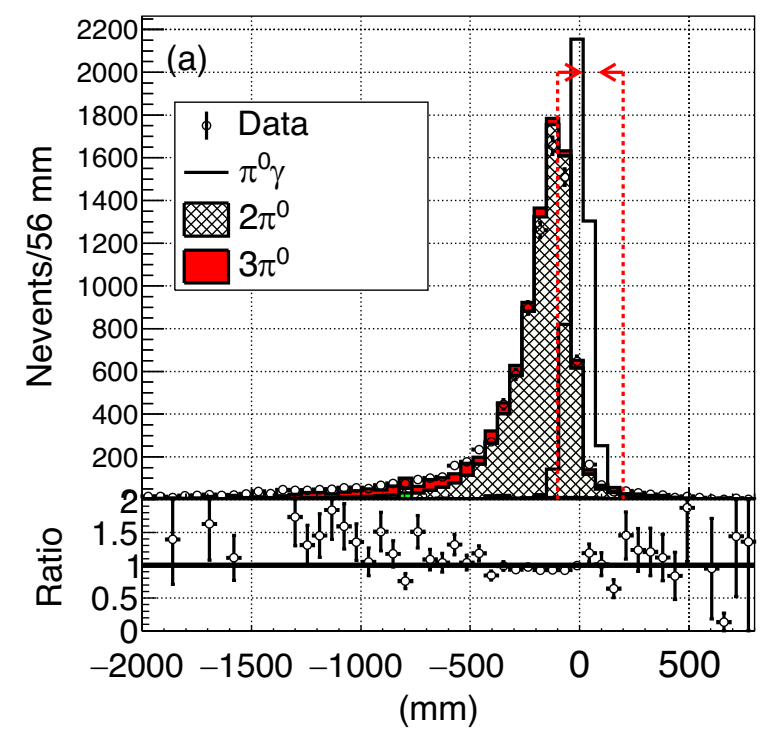

(a) $\Delta z_{\mathrm{vtx}}$

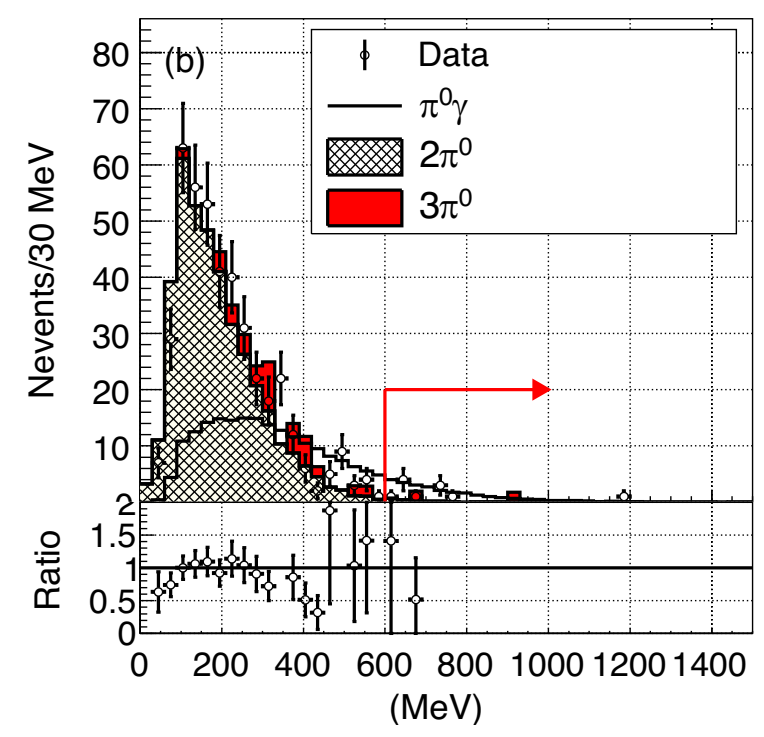

(b) $E_{\gamma}^{\min }$

FIG. 2. (a) Distribution of the two vertex displacement $\left(\Delta z_{\mathrm{vtx}}\right)$ after imposing cuts on the shape $\chi^{2}, \gamma / n$ separation with NN, $K_{L}$ direction, and all the veto counters. (b) Distribution of the minimum energy of three $\gamma$ 's $\left(E_{\gamma}^{\min }\right)$ after imposing the same cuts as (a) and $490 \mathrm{MeV}<M_{\pi^{0} \gamma}<520 \mathrm{MeV}$. Points show data, while histograms of $K_{L} \rightarrow 2 \pi^{0}$ (hatched), $K_{L} \rightarrow 3 \pi^{0}$ (solid), and $K_{L} \rightarrow \pi^{0} \gamma$ (empty), show MC. The histogram for the $K_{L} \rightarrow \pi^{0} \gamma$ decay was obtained assuming $\mathcal{B}\left(K_{L} \rightarrow \pi^{0} \gamma\right)=\mathcal{B}\left(K_{L} \rightarrow 2 \pi^{0}\right) \times 0.01=8.64 \times 10^{-6}$ [22]. Red arrows represent the cuts of $-100 \mathrm{~mm}<\Delta z_{\mathrm{vtx}}<$ $200 \mathrm{~mm} \mathrm{(a)} \mathrm{and} E_{\gamma}^{\min }>600 \mathrm{MeV}$ (b). The bottom regions in both panels show the ratio of data and MC events for each histogram bin. For both figures, the events of data in SR were excluded.

accidental hit. We produced a MC sample and confirmed that the $\Delta z_{\mathrm{vtx}}$ requirement removed all the events in the SR even without imposing various cuts: shape $\chi^{2}, K_{L}$ direction, $\gamma / n$ separation with $\mathrm{NN}$, and $E_{\min }$. We thus set the upper limit to be 0.02 at the $68 \%$ C.L., assuming Poisson distribution.

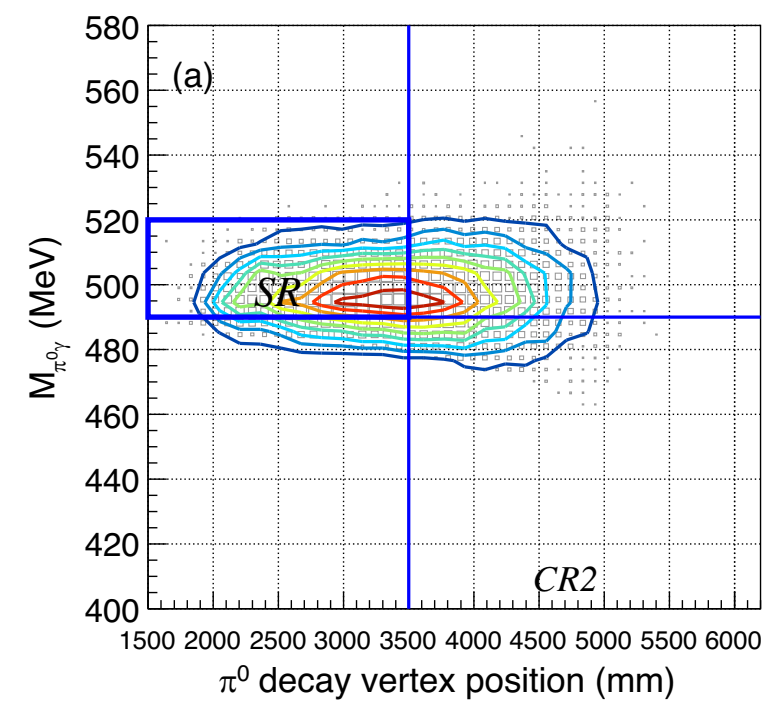

(a) $K_{L} \rightarrow \pi^{0} \gamma$

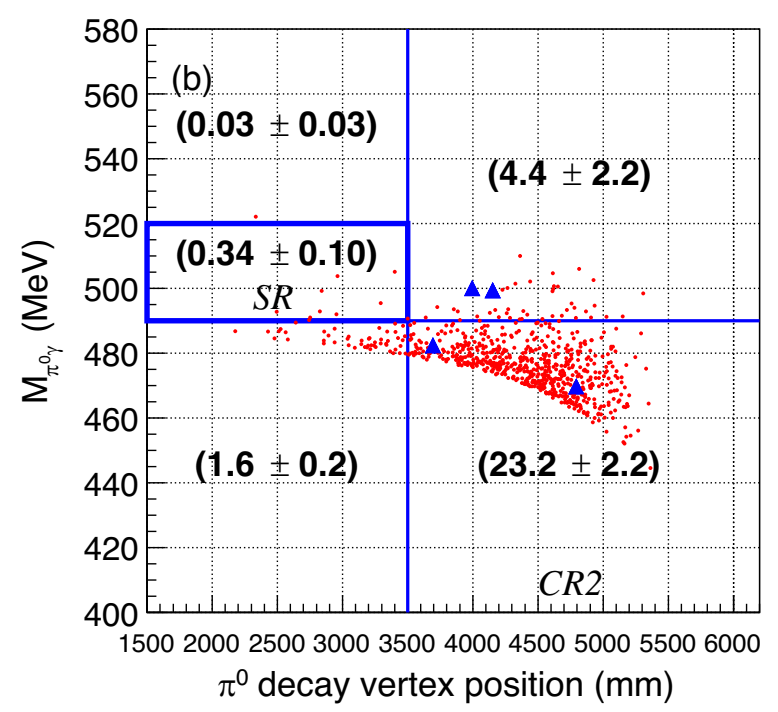

(b) Backgrounds

FIG. 3. Reconstructed $\pi^{0} \gamma$ invariant mass $\left(M_{\pi^{0} \gamma}\right)$ versus $\pi^{0}$ decay point $\left(z_{\mathrm{vtx}}^{\pi^{0}}\right)$ plot by the MC simulation with all cuts imposed on (a) $K_{L} \rightarrow \pi^{0} \gamma$ and (b) background events. The left middle region enclosed by the thick rectangle is the SR and the bottom right is the CR2. The contours in (a) are linear in arbitrary scale. The peak and width ( $\sigma$ of the Gaussian distribution) of the $M_{\pi^{0} \gamma}$ distribution are 497 and $9.4 \mathrm{MeV}$, respectively. In (b), the circular and triangular markers represent $K_{L} \rightarrow 2 \pi^{0}$ and $K_{L} \rightarrow 3 \pi^{0}$ decays, respectively. The markers of $K_{L} \rightarrow 2 \pi^{0}$ and $K_{L} \rightarrow 3 \pi^{0}$ correspond to 0.03 and 1.5 data events, respectively. The numbers in parentheses represent the predicted number of events by the MC simulation.

The $K_{L} \rightarrow \pi^{0} \gamma \gamma$ decay mode could be a background to $K_{L} \rightarrow \pi^{0} \gamma$ if one of the $\gamma$ energies was soft in the laboratory frame. However, due to its small branching fraction of $\mathcal{B}\left(K_{L} \rightarrow \pi^{0} \gamma \gamma\right)=1.27 \times 10^{-6}$ [22], its contribution was negligible.

We also studied the contributions from $K_{L}$ decays with charged particles in the final state. The cut on CV hits 
TABLE I. Acceptances of the $K_{L} \rightarrow \pi^{0} \gamma, K_{L} \rightarrow 2 \pi^{0}$, and $K_{L} \rightarrow 3 \pi^{0}$ decays at each step of event selection.

\begin{tabular}{llccr}
\hline \hline Index & \multicolumn{1}{c}{ Selection } & $K_{L} \rightarrow \pi^{0} \gamma$ & $K_{L} \rightarrow 2 \pi^{0}$ & $K_{L} \rightarrow 3 \pi^{0}$ \\
\hline 1 & $K_{L}$ decay $^{\text {a }}$ & $9 \%$ & $9 \%$ & $9 \%$ \\
2 & Geometry and trigger $_{3}$ & $2.2 \times 10^{-3}$ & $3.1 \times 10^{-4}$ & $1.5 \times 10^{-5}$ \\
3 & Shape $\chi^{2}$ of clusters & $2.0 \times 10^{-3}$ & $2.4 \times 10^{-4}$ & $1.1 \times 10^{-6}$ \\
4 & $x y$ position of clusters & $1.9 \times 10^{-3}$ & $2.2 \times 10^{-4}$ & $9.7 \times 10^{-7}$ \\
5 & $K_{L}$ direction & $1.8 \times 10^{-3}$ & $2.0 \times 10^{-4}$ & $5.8 \times 10^{-7}$ \\
6 & Veto & $7.1 \times 10^{-4}$ & $1.9 \times 10^{-5}$ & $1.5 \times 10^{-8}$ \\
7 & Separation of $\gamma / n$ with NN & $5.1 \times 10^{-4}$ & $1.3 \times 10^{-5}$ & $8.2 \times 10^{-9}$ \\
8 & $\Delta z_{v t x}$ & $4.9 \times 10^{-4}$ & $4.2 \times 10^{-6}$ & $9.1 \times 10^{-10}$ \\
9 & $E_{\gamma}^{\min }>300 \mathrm{MeV}$ & $2.4 \times 10^{-4}$ & $1.0 \times 10^{-6}$ & $4.4 \times 10^{-10}$ \\
10 & $E_{\gamma}^{\min }>600 \mathrm{MeV}$ & $5.0 \times 10^{-5}$ & $4.1 \times 10^{-8}$ & $4.7 \times 10^{-11}$ \\
11 & $\mathrm{CR} 2, E_{\gamma}^{\min }>300 \mathrm{MeV}$ & $\ldots$ & $8.9 \times 10^{-7}$ & $1.2 \times 10^{-10}$ \\
12 & $\mathrm{SR}, E_{\gamma}^{\min }>600 \mathrm{MeV}$ & $(2.11 \pm 0.03) \times 10^{-5}$ & $(5.6 \pm 1.8) \times 10^{-10}$ & $\cdots$ \\
\hline \hline
\end{tabular}

${ }^{a}$ A probability that $K_{L}$ decay occurs in the SR.

suppressed the contributions in the SR to be less than 0.04 events at the $68 \%$ C.L.

The total number of background events and its uncertainty in the SR only includes background estimates with central values because all of the upper limits came from the limited statistics of MC samples. On the other hand, if we conservatively consider the contributions of all the sources, the upper limit of the number of backgrounds in the SR was 1.0 at the $68 \%$ C.L.

The branching fraction of the signal was measured using the numbers of events in the SR and CR2 as

$$
=\frac{\mathcal{B}\left(K_{L} \rightarrow \pi^{0} \gamma\right)=N_{\mathrm{obs}}^{\mathrm{SR}} \cdot S E S}{N_{\mathrm{obs}}^{\mathrm{SR} 2}} \cdot \frac{\mathcal{B}\left(K_{L} \rightarrow 2 \pi^{0}\right) \epsilon_{2 \pi^{0}}^{\mathrm{CR} 2}+\mathcal{B}\left(K_{L} \rightarrow 3 \pi^{0}\right) \epsilon_{3 \pi^{0}}^{\mathrm{CR} 2}}{\epsilon_{\pi^{0} \gamma}^{\mathrm{SR}}},
$$

where $N_{\mathrm{obs}}^{\mathrm{SR}}$ is the number of observed events in the SR, SES is the single event sensitivity of the $K_{L} \rightarrow \pi^{0} \gamma$ decay, $N_{\mathrm{obs}}^{\mathrm{CR} 2}=528$ is the number of the events in the CR2 (under

TABLE II. Expected numbers of backgrounds in the signal region. The upper limits are at $68 \%$ C.L.

\begin{tabular}{lc}
\hline \hline Source & Number of events \\
\hline$K_{L} \rightarrow 2 \pi^{0}$ & $0.32 \pm 0.10$ \\
$K_{L} \rightarrow 3 \pi^{0}$ & $<0.5$ \\
$K_{L} \rightarrow 2 \gamma$ & $<0.06$ \\
Neutron & $<0.02$ \\
$K_{L} \rightarrow \pi^{0} \gamma \gamma$ & $0.020 \pm 0.002$ \\
Other $K_{L}$ decays & $<0.04$ \\
Total & $0.34 \pm 0.10(<1.0)^{\mathrm{a}}$ \\
\hline \hline
\end{tabular}

${ }^{\text {a }}$ See the main text for this calculation. The total number of events and its uncertainty in the SR only include background estimates with central values because all of the upper limits came from the limited statistics of MC samples. If the contributions of all the sources are assumed, the upper limit of the number of backgrounds in the SR is 1.0 at $68 \%$ C.L. the condition of $\left.E_{\gamma}^{\min }>300 \mathrm{MeV}\right), \epsilon_{\pi^{0} \gamma}^{S R}=2.1 \times 10^{-5}$, $\epsilon_{2 \pi^{0}}^{C R 2}=8.9 \times 10^{-7}$, and $\epsilon_{3 \pi^{0}}^{C R 2}=1.2 \times 10^{-10}$ are the acceptances obtained by the MC of the $K_{L} \rightarrow \pi^{0} \gamma, K_{L} \rightarrow 2 \pi^{0}$, and $K_{L} \rightarrow 3 \pi^{0}$ decays in each region, respectively, and $\mathcal{B}\left(K_{L} \rightarrow 2 \pi^{0}\right)=8.64 \times 10^{-4}$ and $\mathcal{B}\left(K_{L} \rightarrow 3 \pi^{0}\right)=19.5 \%$ are the branching fraction of the $K_{L} \rightarrow 2 \pi^{0}$ and $K_{L} \rightarrow 3 \pi^{0}$ decays, respectively [22]. The obtained $S E S$ was $\left(7.1 \pm 0.3_{\text {stat }} \pm 1.6_{\text {syst }}\right) \times 10^{-8}$, where the first and second uncertainties are statistical and systematic, respectively.

The various sources of uncertainties on SES are summarized in Table III. As Eq. (2) shows, systematic uncertainties due to common bias between the $K_{L} \rightarrow$ $\pi^{0} \gamma$ and $K_{L} \rightarrow 2 \pi^{0}$ decays cancel $\left(K_{L} \rightarrow 3 \pi^{0}\right.$ contribution is only $3 \%$ ). Thus, we used the events in the CR2 to conservatively evaluate the acceptances of off-line and on-line vetoes, common kinematic selection, on-line cluster counting, geometry, and reconstruction. Kinematic selections which were not considered to be common were $E_{\gamma}^{\mathrm{min}}$, $\Delta z_{\mathrm{vtx}}$ and the acceptance being inside of the SR. However, the acceptances of these cuts for the $K_{L} \rightarrow 2 \pi^{0}$ decay are

TABLE III. Summary of uncertainties in the single event sensitivity.

\begin{tabular}{lc}
\hline \hline Source & Uncertainty [\%] \\
\hline Off-line veto & 17 \\
Kinematic selection & 12 \\
On-line veto & 6.4 \\
On-line cluster counting & 1.8 \\
Shape $\chi^{2}$ and $\gamma / n$ separation with NN & 1.5 \\
Geometrical & 1.5 \\
Clustering & 1.0 \\
Reconstruction & 0.3 \\
$\mathcal{B}\left(K_{L} \rightarrow 2 \pi^{0}\right)$ & 0.6 \\
Statistics for normalization & 4.4 \\
Total & $4.4_{\text {stat }} \oplus 22_{\text {syst }}$ \\
\hline \hline
\end{tabular}


much smaller than the $K_{L} \rightarrow \pi^{0} \gamma$ decay, and the evaluation of the uncertainty of $S E S$ by the $K_{L} \rightarrow 2 \pi^{0}$ decay is also conservative. For this reason, we evaluated their uncertainties using the events in the CR2 by the loosening $E_{\gamma}^{\min }$ threshold down to $300 \mathrm{MeV}$. Shape $\chi^{2}, \gamma / n$ separation with $\mathrm{NN}$, and clustering, were evaluated using a different control sample which is dominated by the $K_{L} \rightarrow 3 \pi^{0}$ decays.

The largest contribution came from the discrepancies of the off-line veto acceptances between data and MC. The systematic uncertainty of a given off-line cut was calculated using a double ratio:

$$
r=\frac{n_{\text {data }} / \bar{n}_{\text {data }}}{n_{\mathrm{MC}} / \bar{n}_{\mathrm{MC}}}
$$

where $n_{\mathrm{data}, \mathrm{MC}}$ are the numbers of events after imposing all the vetoes, and $\bar{n}_{\text {data,MC }}$ are the corresponding numbers when one of the vetoes was removed. The deviation of $r$ from 1 was the systematic uncertainty from the off-line veto. The quadratic sum of all the vetoes was the total systematic uncertainty due to off-line veto. The second largest effect came from the systematic uncertainty of the kinematic selection described before. Similarly to the offline veto cuts, we relaxed one of the kinematic cuts and compared the double ratio between data and MC. This uncertainty was mainly caused by the limited statistics of data used for this evaluation. The third largest source of the systematic uncertainty was from the on-line veto. This was estimated using data triggered without imposing on-line vetoes while keeping the information on on-line trigger decision. This uncertainty was mainly due to the intentionally loosened off-line veto energy threshold of CV set to minimize the acceptance loss from the accidental hits. As a result, the on-line threshold was close to that of the offline threshold. Uncertainties from on-line cluster counting, cluster-shape discrimination, geometrical acceptance, clustering, and reconstruction were smaller than those from the three aforementioned sources. The uncertainty of the branching fraction of $K_{L} \rightarrow 2 \pi^{0}$ was taken from the PDG value [22]. The total statistical and systematic uncertainties were $4.4 \%$ and $22 \%$, respectively.

After determining the cuts described above, we unmasked the SR and observed no candidate events, as shown in Fig. 4. The discrepancy between the number of observed events and the MC simulation in the upper right region could be explained by the limited statistics of the simulated $K_{L} \rightarrow 3 \pi^{0}$ decay sample. In fact, when we loosened the cut on the minimum photon energy $\left(E_{\gamma}^{\mathrm{min}}\right)$ from 600 to $300 \mathrm{MeV}$, the contribution from the $K_{L} \rightarrow 3 \pi^{0}$ and $K_{L} \rightarrow 2 \pi^{0}$ decays by the MC simulation increased to $41 \pm 7$ and $47 \pm 1$, respectively, in which uncertainties were statistical only, and the sum of them was consistent with the observation of 96 events. Taking into account the systematic uncertainty of SES [24], the upper limit was set

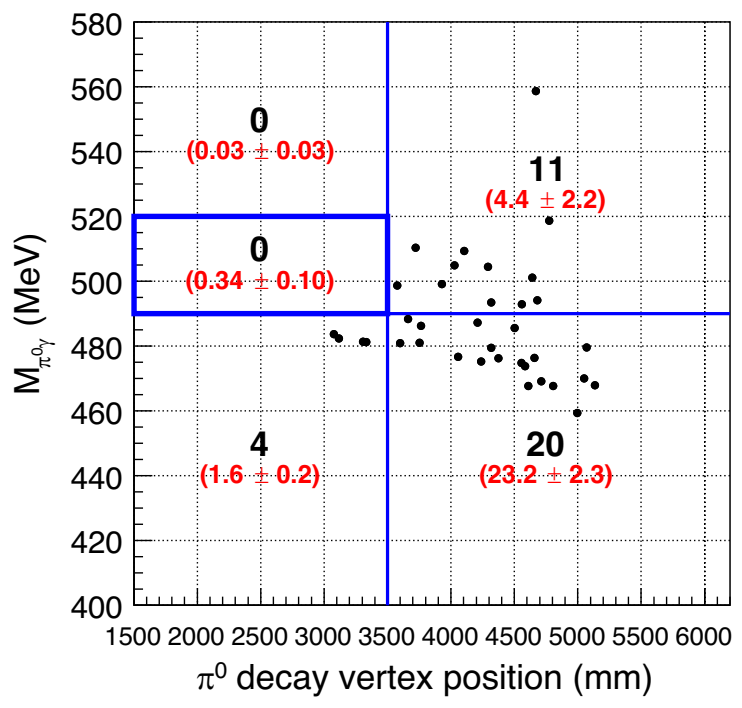

FIG. 4. Reconstructed $\pi^{0} \gamma$ invariant mass $\left(M_{\pi^{0} \gamma}\right)$ versus $\pi^{0}$ decay point $\left(z_{\mathrm{vtx}}^{\pi^{0}}\right)$ plot with all cuts imposed on data. Values are the numbers of the observed events and lower ones (in parentheses) are predictions by the MC simulation.

to $\mathcal{B}\left(K_{L} \rightarrow \pi^{0} \gamma\right)<1.7 \times 10^{-7}$ at the $90 \%$ C.L. This is the first experimental upper limit set on the $K_{L} \rightarrow \pi^{0} \gamma$ decay.

In conclusion, we searched for the $K_{L} \rightarrow \pi^{0} \gamma$ decay, which is forbidden by Lorentz invariance and gauge invariance, for the first time. From the data collected between 2016 and 2018, we observed no candidate events in the signal region. The first upper limit of the branching fraction of the $K_{L} \rightarrow \pi^{0} \gamma$ decay is $1.7 \times 10^{-7}$ at the $90 \%$ confidence level.

\section{ACKNOWLEDGMENTS}

We would like to express our gratitude to all members of the J-PARC Accelerator and Hadron Experimental Facility groups for their support. We also thank the KEK Computing Research Center for KEKCC and the National Institute of Information for SINET4. This work was supported by the Ministry of Education, Culture, Sports, Science, and Technology (MEXT) of Japan and the Japan Society for the Promotion of Science (JSPS) under the MEXT KAKENHI Grant No. JP18071006, the JSPS KAKENHI Grants No. JP23224007 and No. JP16H06343, by the research fellowship program for postdoctoral scientists No. 17J02178, and through the Japan-U.S. Cooperative Research Program in High Energy Physics; the U.S. Department of Energy, Office of Science, Office of High Energy Physics, under Awards No. DE-SC0006497, No. DE-SC0007859, and No. DESC0009798; the Ministry of Education and the Ministry of Science and Technology in Taiwan under Grants No. 1042112-M-002-021, No. 105-2112-M-002-013 and No. 1062112-M-002-016; and the National Research Foundation of Korea (2017R1A2B2011334 and 2019R1A2C1084552). 
[1] S. Oneda, S. Sasaki, and S. Ozaki, Prog. Theor. Phys. 5, 165 (1950).

[2] S. Coleman and S. L. Glashow, Phys. Rev. D 59, 116008 (1999).

[3] Y. Michimura, N. Matsumoto, N. Ohmae, W. Kokuyama, Y. Aso, M. Ando, and K. Tsubono, Phys. Rev. Lett. 110, 200401 (2013).

[4] M. E. Tobar, P. Wolf, S. Bize, G. Santarelli, and V. Flambaum, Phys. Rev. D 81, 022003 (2010).

[5] B. Melić, K. P. Kumerički, and J. Trampetić, Phys. Rev. D 72, 057502 (2005).

[6] A. V. Artamonov et al., Phys. Lett. B 623, 192 (2005).

[7] T. Shimogawa, Nucl. Instrum. Methods Phys. Res., Sect. A 623, 585 (2010).

[8] A. J. Buras, D. Buttazzo, J. Girrbach-Noe, and R. Knegjens, J. High Energy Phys. 11 (2015) 033.

[9] E. Iwai et al., Nucl. Instrum. Methods Phys. Res., Sect. A 786, 135 (2015).

[10] T. Masuda et al., Nucl. Instrum. Methods Phys. Res., Sect. A 746, 11 (2014).

[11] R. Murayama, M. Togawa et al., Nucl. Instrum. Methods Phys. Res., Sect. A 953, 163255 (2020).

[12] D. Naito et al., Prog. Theor. Exp. Phys. 2016, 023 C01 (2016).
[13] Y. Maeda et al., Prog. Theor. Exp. Phys. 2015, 63H01 (2015).

[14] J. K. Ahn et al., Prog. Theor. Exp. Phys. 2017, 021C01 (2017).

[15] J. K. Ahn et al., Phys. Rev. Lett. 122, 021802 (2019).

[16] C. Lin, Y. B. Hsiung, Y. W. Wah, Y. C. Tung, Y. T. Luo, and M. Bogdan, J. Phys. Conf. Ser. 1526, 012034 (2020).

[17] T. Masuda et al., Prog. Theor. Exp. Phys. 2016, $013 \mathrm{C} 03$ (2016).

[18] S. Agostinelli et al., Nucl. Instrum. Methods Phys. Res., Sect. A 506, 250 (2003).

[19] J. Allison et al., IEEE Trans. Nucl. Sci. 53, 270 (2006).

[20] J. Allison et al., Nucl. Instrum. Methods Phys. Res., Sect. A 835, 186 (2016).

[21] A. Hoecker et al., Proc. Sci., ACAT2007 (2007) 040.

[22] M. Tanabashi et al. (Particle Data Group), Phys. Rev. D 98, 030001 (2018).

[23] For the $K_{L} \rightarrow \pi^{0} \gamma$ decay, $M_{01}^{2}+M_{02}^{2}=M_{K_{L}}^{2}-M_{\pi^{0}}^{2}=$ $(479 \mathrm{MeV})^{2}$. This value becomes smaller when the $K_{L} \rightarrow$ $2 \pi^{0}$ decay makes a three cluster event with missing either $\gamma$, whereas this value can become larger when the $K_{L} \rightarrow 3 \pi^{0}$ decay makes a three cluster event with fusion clusters.

[24] R. D. Cousins and V. L. Highland, Nucl. Instrum. Methods Phys. Res., Sect. A 320, 331 (1992). 